\title{
IDENTIFICATION OF THE HARD X-RAY PULSE IN THE FLARE OF SEPTEMBER 11-12, 1968
}

\author{
J. VORPAHL \\ Space Science Laboratory, University of California at Berkeley, Calif., U.S.A. \\ and \\ H. ZIRIN \\ Mount Wilson and Palomar Observatories, Carnegie Institution of Washington, \\ California Institute of Technology, Pasadena, Calif., U.S.A.
}

(Received 16 October, 1969)

Abstract. A hard X-ray pulse in the 11-12 September 1968 flare is identified with the formation of a
brilliant kernel. Each stage in the X-ray event corresponds to a definite phase in flare development.

Kane (1969) has pointed out the existence of two components in many X-ray flares: a sharp spike with a hard spectrum, followed by a more gradual rise. Frost (1969) has reported hard X-ray spikes that do not even appear at lower energies, although a burst of longer duration is seen in the lower channels. An unusually fine event photographed by William Ingham with the Caltech photoheliograph allows us to identify the sources of these X-ray components by comparison of the optical and $\mathrm{X}$-ray data.

McMath plage 658 was the return of plage 593, in which many flares were observed during the previous disk passage. Following the undistinguished-looking preceding spots were parallel regions of following and preceding polarity separated by a filament. There are no magnetograms available for the day, but the principles of the chromospheric magnetograph (Veeder and Zirin, 1969) permit us to deduce the field structure. Figure 1 shows a series of prints of the development of the flare (S top, E right); we can see the bright region of following polarity $S$ of the filament and even penetrating under it; thus, the neutral magnetic line on the surface lies $\mathrm{N}$ of the prominence. The 'imbedded prominence' has long been recognized as an unstable situation. In addition, following polarity here precedes preceding, again unstable. Finally, the flare occurs around 0000 UT, a time at which one of us (Zirin, 1969) found that large flares frequently occur.

Figure 2 (E top, $\mathrm{N}$ right) is a darker version of Figure 1, to bring out the development of the bright kernel of the flare, Figures 1 and 2 should be compared with Figure 3, which gives the X-ray record obtained by the University of California (Berkeley) X-ray experiment on OGO-5.

The X-ray record shows an increase beginning at 235030 , principally in the lowest channel, a sharp spike in the second and third channels beginning 235730 , and a slow rise to a broader maximum at 0019 .

The films show the following phenomena, some of which may be seen on Figures 

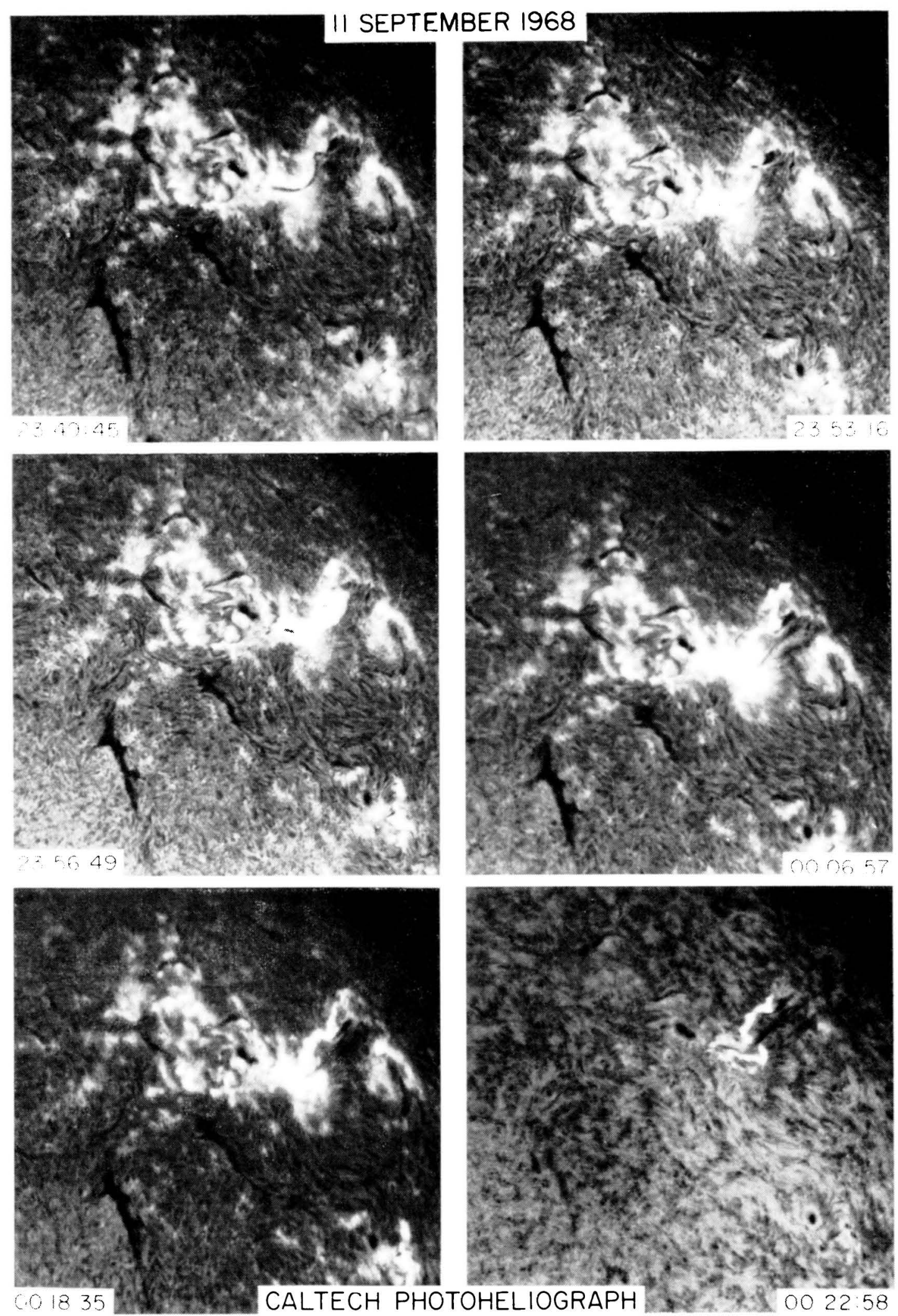

Fig. 1. Sequence of the flare development photographed in $\mathrm{H} \alpha$ with the Caltech Photoheliograph (S top, E right). The last frame is at $\mathrm{H} \alpha-\frac{1}{2} \AA$. 


\section{CALTECH PHOTOHELIOGRAPH}

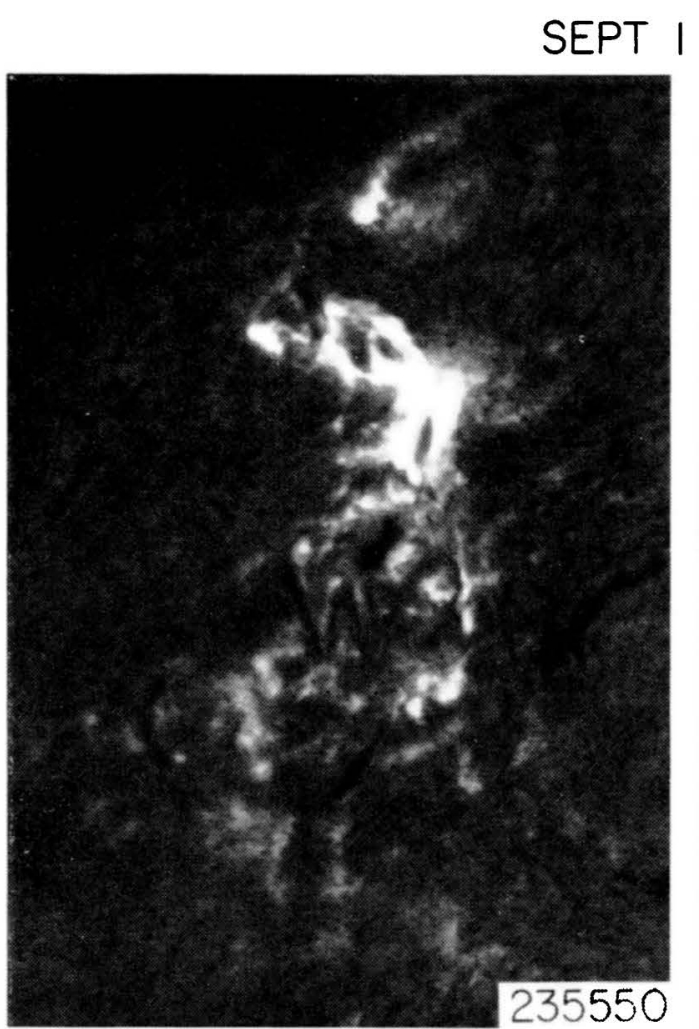

1,1968
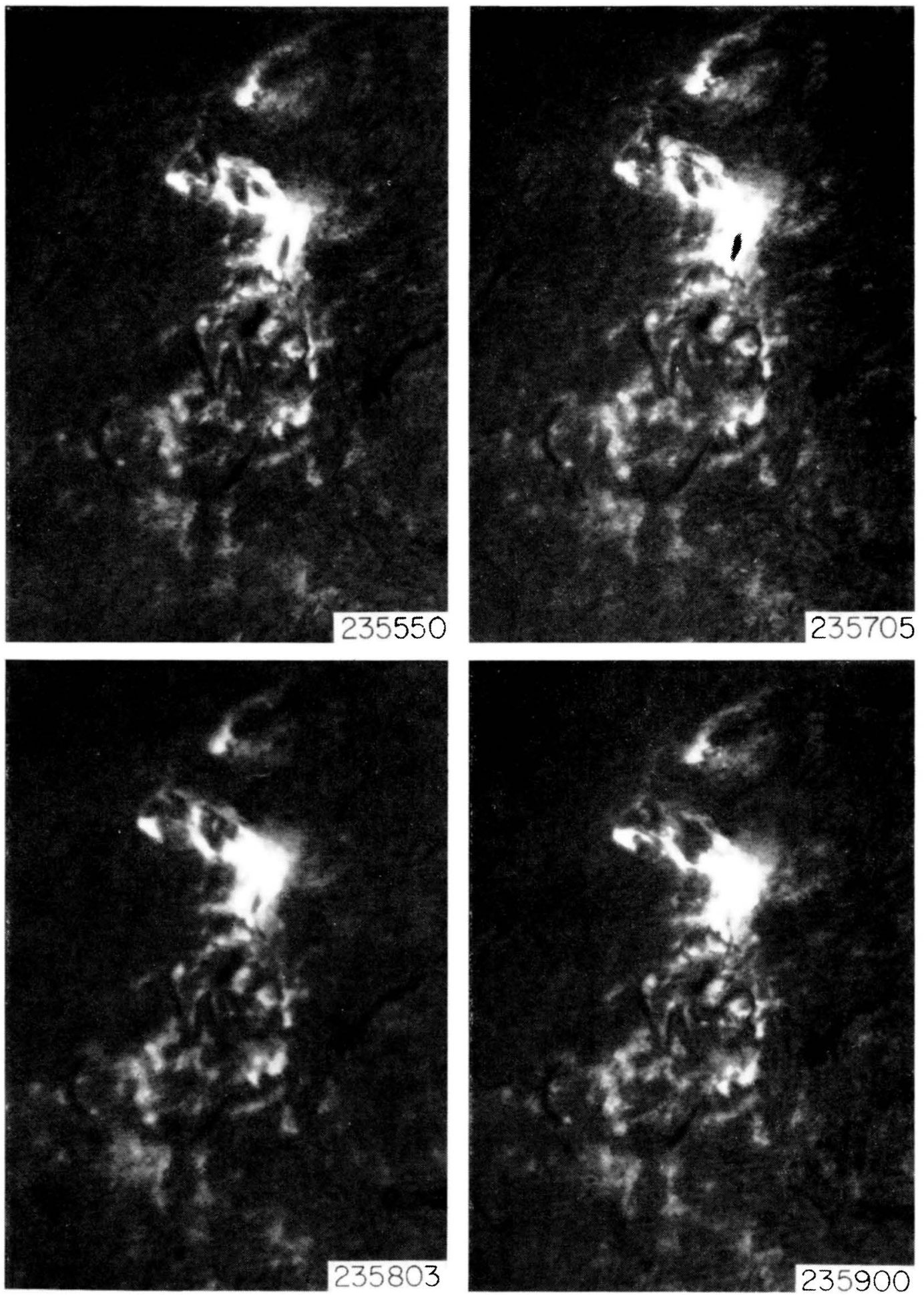

Fig. 2. A dark print of the development of the brilliant kernel which coincides with the hard X-ray spike (E top, $\mathrm{N}$ right). 


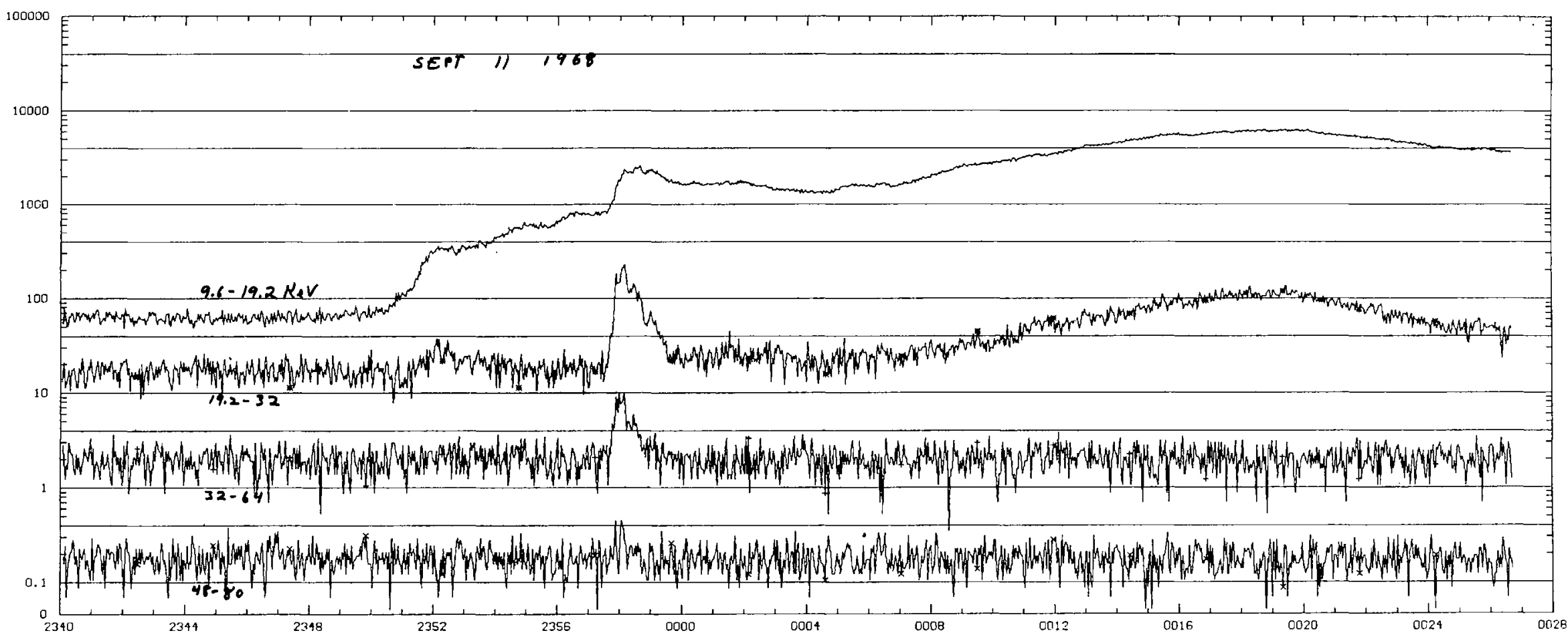

Fig. 3. OGO $5 \mathrm{X}$-ray fluxes. The rates for channel three have been reduced by a factor 0.2 and channel 4 (the highest energy) by 0.02 . 
1 and 2: The prominence was moving outward at 2348, when observations began, but rapid outward motion began at 235040, with a sharp further acceleration at 2352 UT. Both of these times are associated with discontinuities in the X-ray flux, as well as increases in $\mathrm{H} \alpha$ brightness.

At 235730 UT, a great increase in brightness occurred (Figure 2) in a brilliant kernel on the $\mathrm{N}$ side of the filament, in the anomalous area of following field which had penetrated under the prominence. The maximum brightness at 235800 agrees well with the X-ray spike in the higher channels. We can see in the last three frames of Figure 1 how this small area became the source of a tremendous radial outflow of material. In the last frame of Figure 1, we see the bright maximum phase of the flare outlining the edges of the following field region, including the bright kernel (now faded). The channel ratios of the hard spike correspond to brehmsstrahlung from a plasma at a temperature of 120000000 degrees. The value of $N_{\mathrm{e}}^{2} V$ is about $1.5 \times 10^{45}$. The kernel is less than $10^{9} \mathrm{~cm}$ across, so if it is spherical, the density of electrons with this energy is $10^{9}$.

The last two frames of Figure 1 also show the extension of brightness along the surface, which coincides with the low-energy maximum. This would be the reported maximum of the flare area. It is not clear from the films whether this is due to infalling material from the eruption. It is clear that brightening is confined to areas of following polarity only.

One other interesting feature of the flare spray is a sharp right (westward) turn in the outward moving material at 0017 UT. This may have been associated with the type III burst reported at that time.

Table I summarizes the optical correspondence described above.

We see that although the agreement of $\mathrm{H} \alpha$ area with X-ray brightness of area is reasonably good, the relation between $\mathrm{X}$-ray and $\mathrm{H} \alpha$ phenomena is really determined

TABLE I

Optical and X-ray correspondence

\begin{tabular}{lll}
\hline Time & Optical event & X-ray event \\
\hline 235030 & & X-ray increase begins \\
$235040 \pm 10$ & $\begin{array}{l}\text { Acceleration of outward prominence } \\
\text { motion }\end{array}$ & Sharp increase in lowest channel \\
235200 & Sharp acceleration of same & \\
235600 & Kernel brightens & Sharp spike in higher channels \\
235730 & & Maximum of spike \\
235800 & Maximum brightness of kernel & Rise to maximum in lower two channels \\
$0006-0020$ & $\begin{array}{l}\text { Rise to maximum flare area by spread } \\
\text { along surface }\end{array}$ &
\end{tabular}


by the dynamics of the event. Each step in the flare is different, and each produces a different time and energy spectrum.

So far as the dynamics of energetic electron production is concerned, it is interesting that sharp increases in X-ray flux (= acceleration of new electrons) only occur in connection with change, either in brightness or form (usually the former). This means that, whatever the energy storage mechanism, the electrons are only accelerated when changes occur.

Published radio data are sparse. However, Toyokawa reports a series of bursts, again associated with the three phases above: a small burst at 2351 UT was followed by a larger burst at 2358 , followed by a longer-lived burst at 0009 UT.

We conclude that the sharp X-ray spike in the higher channels is connected with the formation of a brilliant kernel in $\mathrm{H} \alpha$; and the main maximum in soft $\mathrm{X}$-rays, with the development of the surface flare in area. The reason for the close association between $\mathrm{H} \alpha$ brightness and hard X-ray flux is not clear; it may be due to the excitation of surrounding, cooler regions by the enormous energy released in the kernel, or it may actually be radiated by the tiny remnant of neutral $\mathrm{H}$ atoms remaining in the kernel.

\section{Acknowledgements}

We wish to express our thanks to Professor Kinsey Anderson for making available for study his data from the OGO-5 Experiment, and to Dr. Sharad Kane for his continued help in understanding and interpreting the records. The University of California, Berkeley Experiment, as well as J. V., is supported by NASA Contract NAS5-9094, and the Cal Tech Photoheliograph, as well as Harold Zirin, by NASA Grant No. NGL 05-002-071, and by NSF Grant No. 1472.

\section{References}

Frost, K.: 1969, paper presented at OSO Workshop, Boulder, Aug. 1969.

Hyder, C.: 1967, Solar Phys. $2,49$.

Kane, S.: 1969, 'Observations of Two Components in Energetic Solar X-ray Bursts', Space Science Lab. preprint.

Veeder, G. and Zirin, H.: 1969, 'The Chromospheric Magnetograph', submitted to Solar Phys. Zirin, H.: 1969, paper presented at AAS Solar Meeting, Pasadena. 